\title{
Distinct substrate specificities of the human tRNA methyltransferases TRMT10A and TRMT10B
}

\author{
NATHAN W. HOWELL, ${ }^{1,2}$ MANASSES JORA, ${ }^{3}$ BENJAMIN F. JEPSON, ${ }^{1,4}$ PATRICK A. LIMBACH, ${ }^{3}$ \\ and JANE E. JACKMAN ${ }^{1,2,4}$ \\ ${ }^{1}$ Center for RNA Biology and Department of Chemistry and Biochemistry, Ohio State University, Columbus, Ohio 43210, USA \\ ${ }^{2}$ Ohio State Biochemistry Program, Ohio State University, Columbus, Ohio 43210, USA \\ ${ }^{3}$ Department of Chemistry and Biochemistry, University of Cincinnati, Cincinnati, Ohio 45221, USA \\ ${ }^{4}$ Molecular, Cellular and Developmental Biology Program, Ohio State University, Columbus, Ohio 43210, USA
}

\begin{abstract}
The tRNA ${ }^{1}{ }^{1}$ g methyltransferase (Trm10) family is conserved throughout Eukarya and Archaea. Despite the presence of a single Trm10 gene in Archaea and most single-celled eukaryotes, metazoans encode up to three homologs of Trm10. Several disease states correlate with a deficiency in the human homolog TRMT10A, despite the presence of another cytoplasmic enzyme, TRMT10B. Here we investigate these phenomena and demonstrate that human TRMT10A (hTRMT10A) and human TRMT10B (hTRMT10B) are not biochemically redundant. In vitro activity assays with purified hTRMT10A and hTRMT10B reveal a robust activity for $h$ TRMT10B as a tRNA ${ }^{A s p}$-specific $m^{1} A_{9}$ methyltransferase and suggest that it is the relevant enzyme responsible for this newly discovered $m^{1} A_{9}$ modification in humans. Moreover, a comparison of the two cytosolic enzymes with multiple tRNA substrates exposes the enzymes' distinct substrate specificities, and suggests that hTRMT10B exhibits a restricted selectivity hitherto unseen in the Trm10 enzyme family. Single-turnover kinetics and tRNA binding assays highlight further differences between the two enzymes and eliminate overall tRNA affinity as a primary determinant of substrate specificity for either enzyme. These results increase our understanding of the important biology of human tRNA modification systems, which can aid in understanding the molecular basis for diseases in which their aberrant function is increasingly implicated.
\end{abstract}

Keywords: tRNA modification; filter binding; methylation assay; SPOUT methyltransferase; N-1 methylation

\section{INTRODUCTION}

Posttranscriptional modification of RNA molecules is a universally conserved process; alteration of nucleotides by the enzymatic addition or rearrangement of chemical groups allows RNA molecules to expand beyond the limits of four nucleotide bases into greater chemical, structural, and functional space (Jackman and Alfonzo 2013; Machnicka et al. 2014). Out of all RNA molecules, tRNA is the most heavily modified, with $\sim 17 \%$ of nucleotides modified in a typical eukaryotic tRNA (Phizicky and Hopper 2010). This extensive posttranscriptional processing represents a heavy investment by the cell to generate a competent pool of tRNA for use in translation. It has increasingly been found that tRNA modifications, and the enzymes that catalyze them, play important roles in human disease (Kirchner and Ignatova 2015), with deficiencies in modification activity so far heavily associated with

Corresponding author: jackman.14@osu.edu

Article is online at http://www.rnajournal.org/cgi/doi/10.1261/rna. 072090.119. mitochondrial and neurological disease phenotypes (Abbott et al. 2014). Despite this medical relevance and their widespread conservation, the function and importance of many tRNA modifications, especially those occurring in the core of the tRNA, has not been completely determined and remains an important question.

The Trm10 tRNA modification enzyme family was discovered in Saccharomyces cerevisiae and catalyzes the addition of a methyl group to the $\mathrm{N}-1$ atom of ninth-position purines $\left(m^{1} R_{9}\right)$ in eukaryotic and archaeal tRNA (Jackman et al. 2003). Trm10 family members have been characterized structurally and biochemically as members of the SPOUT superfamily of methyltransferases (Jackman et al. 2003; Shao et al. 2014; Singh et al. 2018; Krishnamohan and Jackman 2019). Members of the SPOUT family catalyze diverse methylation reactions, modifying distinct

(C) 2019 Howell et al. This article is distributed exclusively by the RNA Society for the first 12 months after the full-issue publication date (see http://rnajournal.cshlp.org/site/misc/terms.xhtml). After 12 months, it is available under a Creative Commons License (Attribution-NonCommercial 4.0 International), as described at http:// creativecommons.org/licenses/by-nc/4.0/. 
atoms and positions in their target nucleotides (Hori 2017; Krishnamohan and Jackman 2019). Despite the diversity of reactions catalyzed, SPOUT family enzymes all possess a common core "SPOUT" domain, characterized by a knotted protein fold (Hori 2017; Krishnamohan and Jackman 2019). All Trm10 enzymes identified to date share overall sequence similarity in this common SPOUT fold, but are flanked by highly dissimilar nonconserved amino- and carboxy-terminal domains (Krishnamohan and Jackman 2017; Singh et al. 2018). Available crystal structures of isolated Trm10 SPOUT domains overlap almost identically, but domains outside of this conserved SPOUT core do not (Shao et al. 2014; Van Laer et al. 2016; Singh et al. 2018).

Previous characterization of Trm10 family members has largely focused on the single homologs found in Archaea and unicellular eukaryotes, and on two of the three human homologs, which exhibit mitochondrial (hTRMT10C) and cytosolic (hTRMT10A) localization, respectively (Kempenaers et al. 2010; Vilardo et al. 2012; Swinehart et al. 2013; Van Laer et al. 2016; Krishnamohan and Jackman 2017; Cosentino et al. 2018). However, a third human homolog (hTRMT10B) is also predicted to exhibit cytosolic localization, but no biological function has yet been attributed to this enzyme. The three human homologs, like all other Trm10 enzymes studied to date, share a SPOUT core that is characterized by significant sequence conservation among active site residues (Krishnamohan and Jackman 2017) and is flanked by distinct amino- and carboxy-terminal domains that are very divergent in terms of overall size and sequence (Jackman et al. 2003; Shao et al. 2014; Singh et al. 2018). The conservation of two distinct cytosolic homologs in humans and other vertebrates has not been explained. Interestingly, deficiency in the activity of hTRMT10A is implicated in multiple disease states, mostly characterized by neurological and glucose metabolic defects, even though the hTRMT10B gene is unaltered in the patients studied (Igoillo-Esteve et al. 2013; Gillis et al. 2014). Although questions about why the absence of hTRMT10A activity contributes to disease remain, it is also unclear why hTRMT10B is apparently unable to replace this important function. Additionally, biochemical activities associated with hTRMT10B have not been clearly demonstrated, and purified hTRMT10B lacked $\mathrm{m}^{1} \mathrm{G}_{9}$ methyltransferase activity with several tested $S$. cerevisiae and human tRNA substrates (Vilardo et al. 2012, 2018).

We hypothesized that the features so far associated with hTRMT10B, including lack of in vitro activity with known substrates for hTRMT10A, and apparent nonredundant biological function, could be explained by distinct substrate specificities for the two enzymes. Here we tested this idea by characterizing biochemical activities associated with hTRMT10A and hTRMT10B across a range of tRNA substrates. Using in vitro tRNA activity and binding assays, we identified a novel function for hTRMT10B in $\mathrm{m}^{1} \mathrm{~A}_{9}$ methylation of a single human tRNA ${ }^{\text {Asp }}$ substrate that is not modified by hTRMT10A, further underscoring the distinct biochemical characteristics that distinguish hTRMT10A and hTRMT10B. Consistent with the biological data, we confirm hTRMT10A as the de facto methyltransferase responsible for all $\mathrm{m}^{1} \mathrm{G}_{9}$ formation so far described on cytosolic tRNA (Vilardo et al. 2012; Cosentino et al. 2018), and demonstrate that hTRMT10B has a much more limited and specific role in tRNA processing in humans.

\section{RESULTS}

\section{hTRMT10A is not functionally redundant with hTRMT10B in vivo in S. cerevisiae}

Unicellular eukaryotes such as $S$. cerevisiae present a simplified opportunity to study the biological functions of Trm10 enzymes, since there is a single Trm10 enzyme that catalyzes all $\mathrm{m}^{1} \mathrm{G}_{9}$ methylation in this organism. We sought to investigate whether the two human cytosolic orthologs hTRMT10A and hTRMT10B function differently in this system by taking advantage of a 5-fluorouracil (5FU) hypersensitivity phenotype that has been previously associated with deletion of $\operatorname{trm} 10$ in $S$. cerevisiae (Gustavsson and Ronne 2008). Plasmids for galactoseinducible expression of the two human Trm10 homologs were transformed into the trm10 10 strain and tested for their ability to rescue the growth-sensitive phenotype (Fig. 1A). As expected, strains expressing $S$. cerevisiae Trm10 (ScTrm10) or hTRMT10A were able to grow at concentrations of 5-FU that suppress the growth of the vector control strain, indicating that hTRMT10A is able to substitute for the required function of ScTrm10 in the presence of 5-FU (Fig. 1A). However, strains containing the hTRMT10B-expressing plasmids grew identically to the empty vector stain, even though western blotting confirmed expression and presence of protein (Fig. 1B), thus supporting the idea that hTRMT10A and hTRMT10B have differing biological functions, resulting in the inability of hTRMT10B to complement the trm104 phenotype.

\section{hTRMT10A and hTRMT10B catalyze methylation of different tRNA substrates}

We questioned whether distinct tRNA substrate specificities of hTRMT10A and hTRMT10B could explain the apparent lack of functional redundancy between these enzymes. To explore this, we cloned, in vitro transcribed, and analyzed several human and $S$. cerevisiae tRNAs as substrates for Trm10-catalyzed methylation. A previously described enzyme assay was used (Swinehart et al. 2013), where methylated enzymatic products are resolved from unmethylated nucleotides after nuclease digestion ( $p^{*} G$ vs. $p^{*} m^{1} G, p^{*} A$ vs. $p^{*} m^{1} A$, respectively) by thin-layer 
A
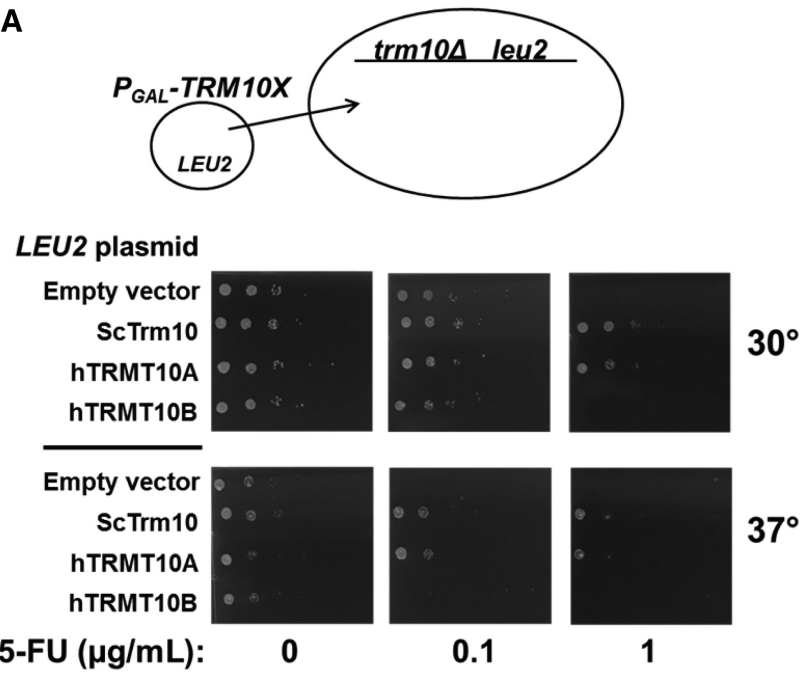

B



FIGURE 1. Human Trm10 orthologs function differently in vivo in $S$. cerevisiae. (A) Complementation assay testing ability of human TRMT10 homologs to complement the S. cerevisiae trm10 $\Delta$ 5-FU hypersensitivity phenotype. The S. cerevisiae trm $10 \Delta$ strain was transformed with LEU2 plasmids expressing various Trm10 homologs under control of a galactose-inducible promoter $\left(P_{G A L}-T R M 10 X\right.$ in schematic). Selected transformants containing either ScTrm10, hTRMT10A, hTRMT10B, or empty vector control plasmid, as indicated, were plated by fivefold serial dilutions onto S-Gal-leu media containing increasing concentrations of $5-\mathrm{FU}(0,0.1$, or $1 \mu \mathrm{g} / \mathrm{mL})$. Images are of plates grown at the indicated temperatures for 2-3 $\mathrm{d}$. (B) Western blot to test for expression of the indicated enzymes in S. cerevisiae trm10 10 strains. Antibodies targeted amino-terminal HA epitopes incorporated into each construct; detected bands matched expected sizes for the indicated enzymes as follows: ScTrm10 (36 kDa), hTRMT10A (41 kDa), hTRMT10B (37 kDa). Tubulin loading control is shown on the lower blot.

chromatography (TLC). Multiple yeast and human tRNAs (Fig. 2; Supplemental Fig. S1; summarized in Table 1) were screened for methylation activity after incubation with purified recombinant hTRMT10A or hTRMT10B. Consistent with previous observations (Vilardo et al. 2012; Cosentino et al. 2018), hTRMT10A displayed robust activity on every tested $\mathrm{G}_{9}$-containing tRNA from either humans or S. cerevisiae, similar to ScTrm10 (Fig. 2A,C; Supplemental Fig. S1). In contrast, hTRMT10B displayed only very weak $\mathrm{m}^{1} \mathrm{G}_{9}$ activity on two substrates (human
tRNA ${ }^{\text {Arg }}$ and tRNA ${ }^{\text {Trp }}$, with no detectible activity on any other $\mathrm{G}_{9}$-containing tRNA (Fig. 2B). This discrepancy between the ability of the two purified human enzymes to catalyze $\mathrm{m}^{1} \mathrm{G}_{9}$ modification is consistent with the lack of apparent functional redundancy suggested by the previous biological data (Igoillo-Esteve et al. 2013; Gillis et al. 2014; Cosentino et al. 2018), and further supports the previously described role of $h$ TRMT10A as the de facto human cytoplasmic $\mathrm{m}^{1} \mathrm{G}_{9}$ methyltransferase (Cosentino et al. 2018).

Due to $h T R M T 10 B^{\prime}$ s relative lack of $m^{1} G_{9}$ activity, as well as the lack of a corresponding enzyme for the recently reported $\mathrm{m}^{1} \mathrm{~A}_{9}$ on cytosolic tRNA ${ }^{\text {Asp }}$ (Clark et al. 2016), we also screened for activity on several Aq-containing tRNAs. We found that hTRMT10B, and not ScTrm10 or hTRMT10A, is indeed capable of catalyzing $\mathrm{m}^{1} \mathrm{~A}_{9}$ methylation on tRNA ${ }^{\text {Asp }}$ in vitro, based on its ability to generate the labeled $\mathrm{p}^{*} \mathrm{~m}^{1} \mathrm{~A}$ product that is also observed in reactions with the known $m^{1} A_{9}$ methyltransferase from Thermococcus kodakarensis (TkTrm10) (Fig. 2D). The inability of ScTrm10 or hTRMT10A to catalyze any detectable $\mathrm{m}^{1} \mathrm{~A}_{9}$ modification is consistent with previous biochemical studies with three other human tRNAs and suggests that hTRMT10B has the sole responsibility for generating the $\mathrm{m}^{1} \mathrm{~A}_{9}$ modification that is found on tRNA ${ }^{\text {Asp }}$ in humans (Vilardo et al. 2012). To confirm the identity of the modification catalyzed by hTRMT10B in the in vitro assays, unlabeled tRNA transcripts were incubated with and without hTRMT10B, and analyzed for the presence of ribonucleoside modifications using high-performance liquid chromatography-tandem mass spectrometry (HPLC-MS/MS). HPLC-MS/MS of nucleosides and oligonucleosides confirmed that the only enzymatic product detected was $\mathrm{m}^{1} \mathrm{~A}$, and that the methylation was at position $A_{9}$ of the tRNA (Fig. 3A; Supplemental Fig. 2). To confirm that the observed methylation activity was indeed due to hTRMT10B, we created hTRMT10B variants with two different inactivating mutations known to disrupt conserved SAM-binding residues G231 and G232 (G207 and G208 in the context of hTRMT10A) (Gillis et al. 2014; Shao et al. 2014; Krishnamohan and Jackman 2017). The single variant (G231R) abolished methylation of tRNA ${ }^{\text {Asp }}$ and tRNA ${ }^{\text {Trp }}$, with some residual (though reduced) activity on tRNA ${ }^{\text {Arg }}$ (Fig. 3B), and the double variant (G231R/ G232R) displayed no methylation activity on any of the three substrates (Fig. 3C).

\section{hTRMT10B exhibits extreme tRNA substrate selectivity in vitro}

ScTrm10 and its human homolog hTRMT10A are known to exhibit considerable promiscuity in terms of in vitro $\mathrm{m}^{1} \mathrm{G}_{9}$ methylation activity, with both enzymes capable of methylating every $\mathrm{G}_{9}$-containing Type I tRNA so far tested using in vitro assays (Vilardo et al. 2012; Swinehart et al. 2013), 
A

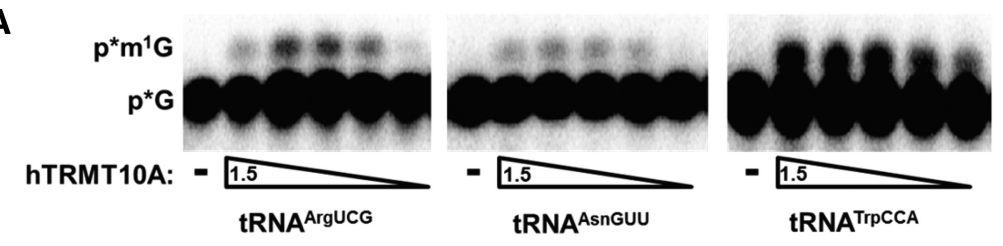

B

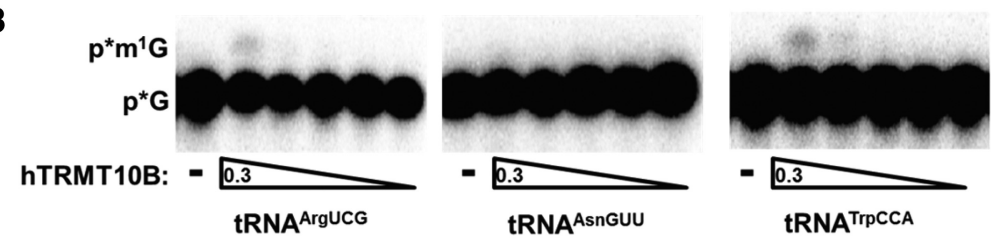

C

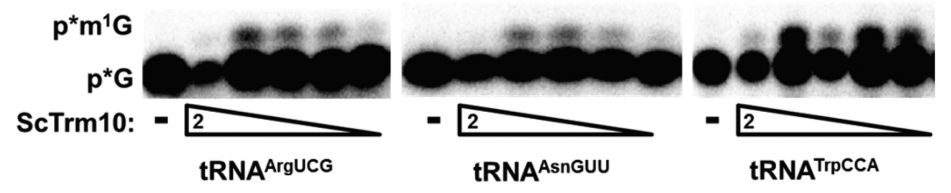

D

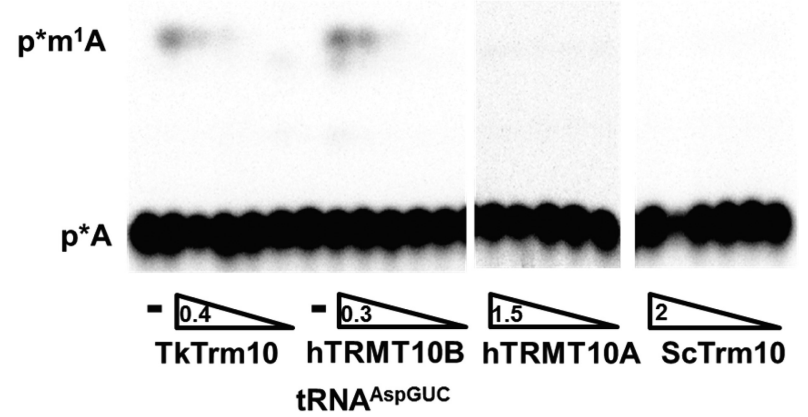

FIGURE 2. Human TRMT10A and TRMT10B exhibit distinct in vitro tRNA substrate specificities. (A) $m^{1} G_{9}$ activity of hTRMT10A on human $G_{9}$-containing tRNAs. Labeled tRNA were created by in vitro transcription in the presence of $\left[\alpha^{32} P\right]-G T P$, resulting in uniform labeling at every $\mathrm{G}$ nucleotide. Labeled tRNA were incubated with fivefold dilutions of the enzyme $(1.5 \mathrm{mg} / \mathrm{mL}$ to $2.4 \mu \mathrm{g} / \mathrm{mL}$, with the highest concentration indicated by the number shown in the wedge representing each titration) or no enzyme (-) for $1 \mathrm{~h}$. Quenched reactions were digested to single nucleotides using P1 nuclease and analyzed by TLC to resolve product $\left(p^{*} m^{1} G\right)$ from remaining unreacted substrate $\left(p^{*} G\right)$. (B) $m^{1} G_{9}$ activity of $h$ TRMT10B on human $\mathrm{G}_{9}$-containing tRNAs. Reactions were performed identically to $A$, except that serial dilutions contained $0.3 \mathrm{mg} / \mathrm{mL}$ to $0.5 \mu \mathrm{g} / \mathrm{mL}$ enzymes, with the highest concentration indicated by the number shown in the wedge representing each titration as above. (C) $\mathrm{m}^{1} \mathrm{G}_{9}$ activity of ScTrm10 on human $G_{9}$-containing tRNAs. Reactions were performed identically to $A$, except that serial dilutions contained $2 \mathrm{mg} / \mathrm{mL}$ to $3 \mu \mathrm{g} / \mathrm{mL}$ enzyme, with highest concentration indicated by the number shown in the wedge representing each titration as above. (D) $m^{1} A_{9}$ activity of hTRMT10A and hTRMT10B, and T. kodakarensis Trm10 positive $\mathrm{m}^{1} \mathrm{~A}_{9}$ control (Krishnamohan et al. 2019), on human tRNA ${ }^{\text {Asp }}$. Uniformly A-labeled in vitro transcript of tRNA ${ }^{\text {Asp }}$ was prepared as described in $B$ above (in transcription reactions containing [ $\left.\alpha^{32} \mathrm{P}\right]-$ ATP), and tested with the same assay. Digestion with nuclease P1 generates $p^{*} m^{1} A$ from methylated product RNA and P*A from unreacted substrate, which are also resolved by TLC.

although it has been observed that ScTrm10 modifies a restricted subset of the $\mathrm{G}_{9}$-containing tRNAs in vivo (Juhling et al. 2009; Swinehart et al. 2013). Both hTRMT10A and ScTrm10 exhibited similar patterns of $\mathrm{m}^{1} \mathrm{G}_{9}$ methylation on all substrates tested in this work, consistent with these previous observations (Table 1). However, hTRMT10B does not exhibit similar promiscuity, as evident from its inability to detectably methylate any of three other $\mathrm{A}_{9}$ containing tRNAs tested using a similar in vitro activity assay with transcripts representing human tRNA ${ }^{\text {Ala }}$ and TRNA $^{\text {Phe }}$, and $S$. cerevisiae tRNA ${ }^{\text {Phe }}$. In these assays, hTRMT10B is only able to methylate tRNA ${ }^{\text {Asp }}$, and shows no detectable methylation on the other three tRNA (Fig. 4A). The reason for the observed lack of hTRMT10B activity is not apparent when comparing either secondary structures of the tested tRNAs (Fig. $4 \mathrm{~B})$ or sequences of $\mathrm{A}_{9}$-containing tRNAs in humans (Fig. 4C; Juhling et al. 2009). The possibility that other yet to be identified substrates for in vitro methylation by hTRMT10B exist cannot be excluded. Nonetheless the substrate selective behavior observed so far for this enzyme places it in stark contrast to other tested Trm10 family members such as ScTrm10 and hTRMT10A, and even the mitochondrial hTRMT10C, which is able to N-1 methylate many tRNAs with $A, G$, and even I at position 9 (Vilardo et al. 2012).

\section{In vitro tRNA substrate specificity patterns exhibited by hTRMT10A and hTRMT10B are not determined by tRNA affinity}

With differences in substrate specificity between hTRMT10A and hTRMT10B established, we assessed whether different methylation patterns could be explained by distinct tRNA binding affinities exhibited by each enzyme. Filter binding assays were used to measure apparent affinities between each purified enzyme and four selected tRNA species, chosen to represent both substrate and nonsubstrate tRNAs for both enzymes. Interestingly, for the four tested tRNAs, we observed no substantial differences in the apparent equilibrium dissociation constant $\left(K_{\mathrm{D}, \mathrm{app}}\right)$ between hTRMT10A and hTRMT10B. These assays revealed only approximately fivefold difference in $K_{D \text {,app }}$ between hTRMT10A's most tightly bound tRNA (tRNA ${ }^{\text {Arg) }}$ and hTRMT10B's most weakly bound tRNA (tRNA ${ }^{\text {Asp) }}$ (Fig. 5). 
TABLE 1. In vitro methylation activity of Trm10 enzymes

\begin{tabular}{|c|c|c|c|c|}
\hline Nucleotide at position 9 & tRNA (anticodon) & ScTrm 10 activity & hTRMT10A activity & hTRMT10B activity \\
\hline \multicolumn{5}{|l|}{ Human tRNAs } \\
\hline \multirow[t]{8}{*}{$\mathrm{G}_{9}$} & Arg (UCG) & +++ & +++ & + \\
\hline & Asn (GUU) & +++ & +++ & - \\
\hline & Gln (CUG) & +++ & +++ & - \\
\hline & Gln (UUG) & +++ & +++ & - \\
\hline & Gly (CCC) & +++ & +++ & - \\
\hline & Gly (GCC) & +++ & +++ & - \\
\hline & iMet (CAU) & +++ & +++ & - \\
\hline & $\operatorname{Trp}(C C A)$ & +++ & +++ & + \\
\hline \multirow[t]{3}{*}{$A_{9}$} & Ala (AGC) & - & - & - \\
\hline & Asp (GUC) & - & - & ++ \\
\hline & Phe (GAA) & $N D^{a}$ & - & - \\
\hline \multicolumn{5}{|l|}{ Yeast tRNAs } \\
\hline \multirow[t]{6}{*}{$\mathrm{G}_{9}$} & Asn (GUU) & +++ & +++ & - \\
\hline & Gly (CCC) & +++ & +++ & - \\
\hline & Gly (GCC) & +++ & +++ & - \\
\hline & Leu (CAA) & - & - & - \\
\hline & $\operatorname{Trp}(\mathrm{CCA})$ & +++ & +++ & - \\
\hline & Val (UAC) & +++ & +++ & - \\
\hline$A_{9}$ & Phe (GAA) & $N D^{a}$ & $N D^{a}$ & - \\
\hline
\end{tabular}

${ }^{a}(N D)$ Not determined as part of this work.

In fact, we found that hTRMT10A and hTRMT10B display slightly lower affinity for substrates upon which they have higher methylation activity (Table 2, discussed below). Taken together, it is clear that apparent affinity of the enzyme for certain tRNA species is not a determinant of catalytic potential, and certainly does not explain the ability of hTRMT10B to preferentially methylate tRNA ${ }^{\text {Asp }}$ over other $A_{9}$-containing tRNAs, since $K_{D, \text { app }}$ for this tRNA is similar to or even greater than that observed for the nonsubstrate tRNA $^{\text {Ala }}$ (Fig. 5). Thus, these data suggest that tRNA binding alone is not the primary determinant of either hTRMT10A or hTRMT10B activity.

\section{hTRMT10A displays higher in vitro catalytic rates than hTRMT10B}

To further quantify differences between hTRMT10A and hTRMT10B, we performed a single-turnover kinetic analysis of each enzyme with representative tRNA substrates, tRNA $^{\text {Arg }}$, tRNA ${ }^{\text {Trp }}$, and tRNA ${ }^{\text {Asp }}$ (tRNA ${ }^{\text {Ala }}$ is not detectably methylated by either enzyme and therefore was not tested) using the same activity assays described above. Observed rates $\left(k_{\text {obs }}\right)$ were determined for each enzyme using saturating concentrations of enzyme, which enables comparison of the rate-determining steps for the methylation step for each substrate, as described previously (Krishnamohan and Jackman 2017). In these assays, hTRMT10A exhibited a significantly higher overall catalytic efficiency than hTRMT10B, with 380-fold and 1100-fold greater $k_{\text {obs }}$ values exhibited for tRNA ${ }^{\text {Arg }}$, and tRNA ${ }^{\text {Trp }}$, respectively (Table 2). The relatively weak $\mathrm{m}^{1} \mathrm{G}_{9}$ methylation activity of hTRMT10B fits with the unique role of hTRMT10A as the cytosolic $\mathrm{m}^{1} \mathrm{G}_{9}$ methyltransferase in humans. The rates of $h$ TRMT10B-catalyzed $m^{1} A_{9}$ methylation on tRNA ${ }^{\text {Asp }}$, although on the low end of catalytic efficiency among other studied Trm10 family members, are nonetheless modestly higher than those observed for $\mathrm{m}^{1} \mathrm{G}_{9}$ methylation. Moreover, the inability of hTRMT10A to catalyze any detectable activity on the tRNA ${ }^{\text {Asp }}$ that is known to be modified with $\mathrm{m}^{1} \mathrm{~A}_{9}$ in human cells (Clark et al. 2016) is consistent with this activity representing the true biological function of hTRMT10B in humans. We note that while these studies were in progress, independent genetic analysis demonstrated that depletion of hTRMT10B in human cells led to complete loss of $\mathrm{m}^{1} \mathrm{~A}_{9}$ modification of human tRNA ${ }^{\text {Asp }}$, confirming this function of the human TRMT10B ortholog in vivo (E Vilardo and W Rossmanith, pers. comm.).

\section{DISCUSSION}

Interest in understanding mechanisms by which tRNA modification enzymes select and modify substrate tRNA has increased as roles for these enzymes in human disease have become more apparent (Abbott et al. 2014; Kirchner and Ignatova 2015). The identification of nearly complete sets of tRNA modification enzymes and their targets in $S$. cerevisiae and E. coli facilitated many early studies focused 

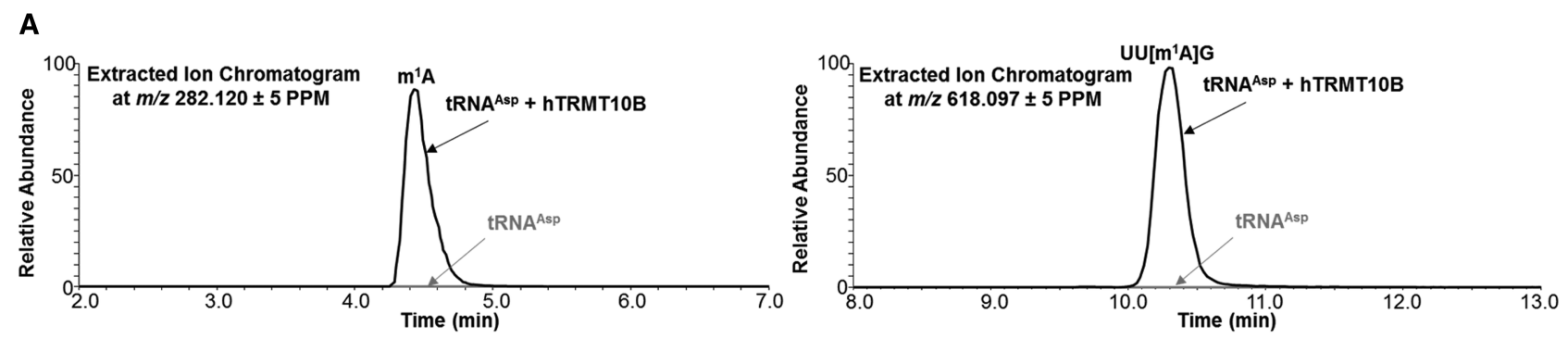

B
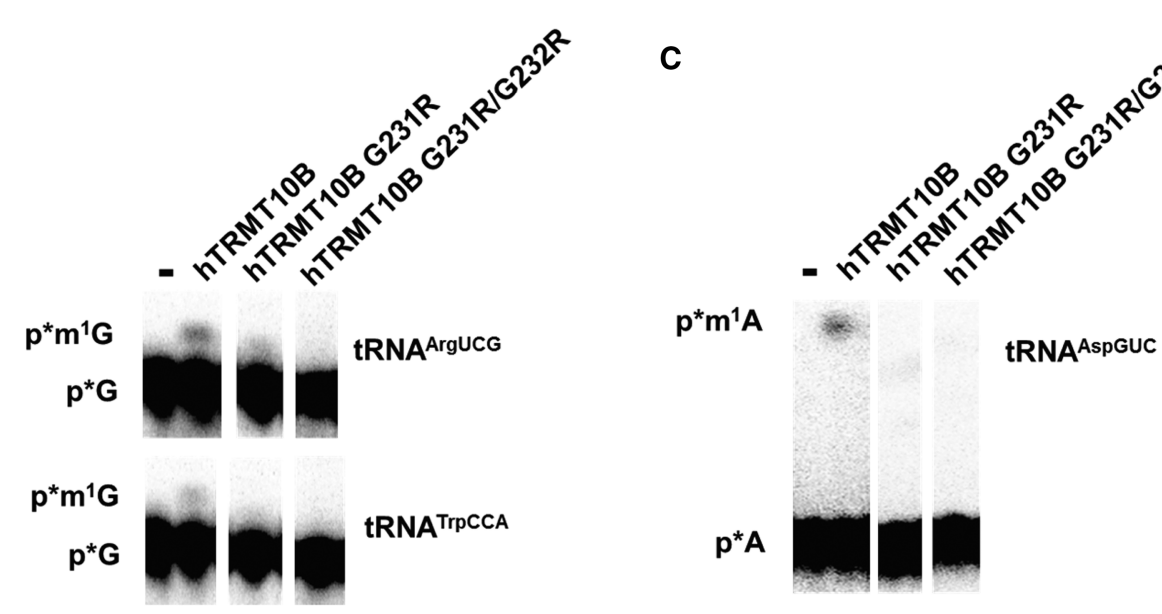

FIGURE 3. Validation of $m^{1} A_{9}$ methylation product catalyzed by hTRMT10B. (A) Extracted ion chromatograms of the nucleoside (left) and the oligonucleotide (right) analyses of tRNA ${ }^{\text {Asp }}$ transcript (with and without hTRMT10B incubation indicated by black and gray arrows, respectively). The nucleoside analysis confirmed that $\mathrm{m}^{1} \mathrm{~A}$ (the only ribonucleoside modification detected) is only observed in the transcript incubated with hTRMT10B. The oligonucleotide analysis revealed the presence of the unique and sequence-specific $U U\left[\mathrm{~m}^{1} \mathrm{~A}\right] \mathrm{G}$ oligonucleotide, confirming $\mathrm{m}^{1} \mathrm{~A}$ at position 9 of tRNA ${ }^{\text {Asp. }}$. Mass spectra information is shown in Supplemental Figure 2. $(B, C)$ Methylation activities of hTRMT10B variants with inactivating mutations in the conserved SAM binding residues. Activity assays were performed with either single variant G231R or double variant G231R/G232R purified enzymes $(0.3$ and $0.2 \mathrm{mg} / \mathrm{mL}$, respectively), with tRNAs uniformly labeled at $\mathrm{G}$ nucleotides $(B)$ or $A$ nucleotides (C), as in Figure 2.

on activities in these organisms, while an understanding of several biologically important human enzymes has lagged (Juhling et al. 2009; Phizicky and Hopper 2010; Jackman and Alfonzo 2013). Similarly, studies of modifications in and around the anticodon loop of tRNA are represented frequently in the literature due to readily observed translational phenotypes associated with loss of many of these types of modifications, whereas studies of modifications in the core of tRNA have lagged (Gu et al. 2014; Endres et al. 2015; Tuorto and Lyko 2016). Here we attempted to address both gaps by characterizing two human tRNA methyltransferases responsible for modifications at ninthposition purine residues.

Seeking to explain the apparent lack of functional redundancy between hTRMT10A and hTRMT10B, we showed that the two enzymes have distinct functionalities, as evidenced by their distinct in vitro substrate specificities, and identify hTRMT10B as the enzyme responsible for the recently discovered (Clark et al. 2016) $\mathrm{m}^{1} \mathrm{~A}_{9}$ modification in tRNA ${ }^{\text {Asp }}$ (Fig. 2). In parallel with the work described here, a genetic approach was used to identify the same tRNA ${ }^{\text {Asp }} \mathrm{m}^{1} \mathrm{~A}_{9}$ modification activity associated with hTRMT10B in HAP1 cells, in agreement with our ob- servations (E Vilardo and W Rossmanith, pers. comm.). So far, out of several studies seeking to characterize modifications found in human tRNA species, tRNA ${ }^{\text {Asp }}$ is the only tRNA demonstrated to contain the $\mathrm{m}^{1} \mathrm{~A}_{9}$ modification (Clark et al. 2016), and thus tRNA ${ }^{\text {Asp }}$ appears to be the single biological target for hTRMT10B activity. It remains unclear why humans or other organisms encode this second cytosolic TRMT10B homolog if its only function is to catalyze modification of a single target tRNA. However, these results suggest that hTRMT10B is not present to simply act in a tissue-specific manner to replace existing hTRMT10A functions, as had been previously suggested (Gillis et al. 2014). Therefore the conservation of TRM10B orthologs throughout vertebrates suggests that the ability to catalyze distinct modifications at position 9 provides some evolutionary advantage (Jackman et al. 2003).

It is interesting that, unlike the other members of the Trm10 family characterized so far, which act relatively nonselectively on various tRNA species that contain the appropriate target nucleotide in vitro (Vilardo et al. 2012; Swinehart et al. 2013; Cosentino et al. 2018), hTRMT10B was not able to modify three other tested tRNA species that contain the $A_{9}$ nucleotide (Fig. 4A). This apparent 
A


FIGURE 4. Human TRMT10B exhibits limited tRNA substrate specificity. (A) In vitro methylation assays were performed with four different $A_{9}$ containing yeast and human tRNAs. Serial dilutions of hTRMT10B $(0.3 \mathrm{mg} / \mathrm{mL}$ to $0.5 \mu \mathrm{g} / \mathrm{mL})$, or no enzyme (-) were incubated with the uniformly

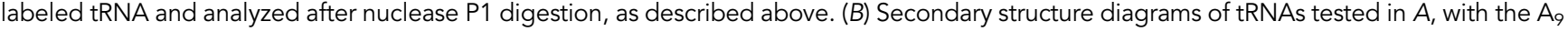
residue highlighted in red for each tRNA. (C) Multiple sequence alignment of $A_{9}$-containing human tRNA genes (Juhling et al. 2009). Alignment was performed using Clustal Omega (Sievers et al. 2011), similar nucleotides are highlighted by shading (using BoxShade), and nucleotides unique to tRNA ${ }^{\text {Asp }}$ among all other $A_{9}$ tRNA are starred and shown in red.

stricter substrate selectivity is another way that hTRMT10B appears to biochemically differ from other Trm10 enzymes. Although the molecular basis for this tRNA-selective modification activity is not known, it may suggest that presence of $\mathrm{m}^{1} \mathrm{~A}_{9}$ could be deleterious if formed on other tRNAs, thus explaining the evolution of more restrictive substrate specificity for this particular family member.
Measurements of in vitro rates of methylation catalyzed by hTRMT10B consistently indicated a significantly lower apparent catalytic efficiency exhibited by the enzyme compared to other Trm10 homologs, including hTRMT10A (Table 2; Krishnamohan and Jackman 2017; Krishnamohan et al. 2019). The relative lack of efficient $m^{1} G_{9}$ methylation catalyzed by hTRMT10B is consistent with the scenario in 


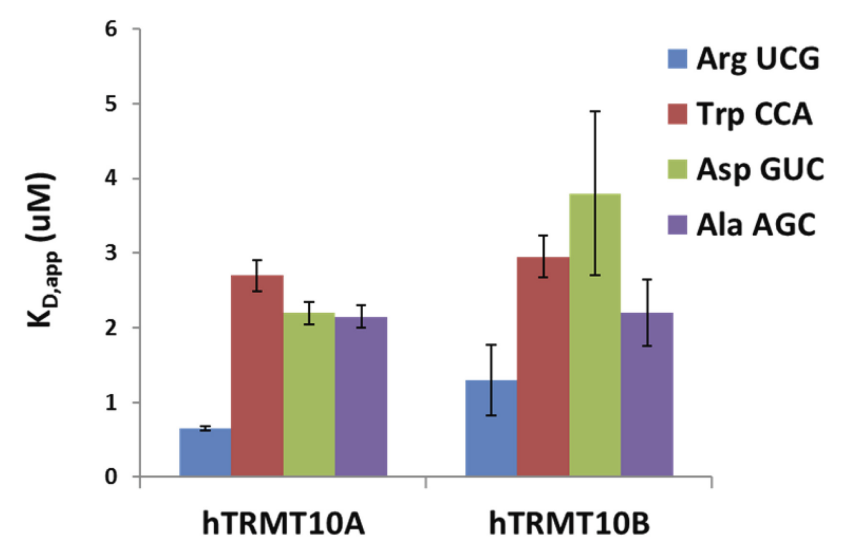

FIGURE 5. Binding affinity does not determine substrate specificity patterns for hTRMT10A and hTRMT10B. Affinities of hTRMT10A and hTRMT10B were determined for each of four different human tRNAs using a double-filter binding assay. The four tRNAs were chosen to represent two $\mathrm{G}_{9}$-containing tRNA (Arg and Trp, modified efficiently by hTRMT10A and much less efficiently by hTRMT10B), and two $A_{9}$-containing tRNAs (Asp and Ala, with Asp modified only by hTRMT10B, and Ala modified by neither enzyme). Apparent $K_{\mathrm{D}}$ values determined by the fit of the data from the binding assay, as described in Materials and Methods, are plotted for each enzyme. Measured $K_{\mathrm{D}, \text { app }}$ for hTRMT10A: $0.65 \mu \mathrm{M}$ (Arg), $2.7 \mu \mathrm{M}$ (Trp), $2.2 \mu \mathrm{M}$ (Asp), and 2.2 $\mu \mathrm{M}$ (Ala), and for hTRMT10B: $1.3 \mu \mathrm{M}$ (Arg), 3.0 $\mu \mathrm{M}$ (Trp), 3.8 $\mu \mathrm{M}$ (Asp), and $2.2 \mu \mathrm{M}$ (Ala). Error bars represent standard error derived from two independent assays.

which $h$ TRMT10A is the biologically relevant $m^{1} G_{9}$ methyltransferase, and with the fact that hTRMT10B expression cannot complement the growth defect associated with trm10 deletion in $S$. cerevisiae, despite its weak ability to catalyze this reaction with at least two tRNA (Fig. 1). However, as hTRMT10B is the only known enzyme in humans that is capable of introducing the tRNA ${ }^{\text {Asp }} \mathrm{m}^{1} \mathrm{~A}_{9}$ modification (Clark et al. 2016), the relatively slow in vitro rates raise questions about the biological sufficiency of this enzyme. Interestingly, a recent analysis of the archaeal bifunctional $\mathrm{m}^{1} \mathrm{~A} / \mathrm{m}^{1} \mathrm{G}$ homolog from Thermococcus kodakarensis ( $T$ kTrm0) revealed significant substrate-dependent differences in rate, with several tRNA species being methylated with 100-fold slower rates than others (Singh et al. 2018; Krishnamohan et al. 2019). Understanding how hTRMT10B specifically interacts with its target tRNA substrate will be important to determine the basis for any role for the identity of the tRNA in dictating overall catalytic efficiency. Nonetheless, the tRNA binding experiments performed here (Fig. 5) suggest that differences in overall tRNA affinity are not able to explain the observed tRNA substrate specificity, and demonstrate the need for further experiments detailing the interaction of hTRMT10B with tRNA.

The lower observed rates of methylation by hTRMT10B could also indicate a dependence on one or more other modifications for efficient activity, and the absence of native tRNA modifications on the in vitro tRNA transcripts tested in the assays may play a role in the observed low catalytic rates. For ScTrm10, efficiency of methylation was somewhat affected by the use of modified tRNA substrates derived from trm $10 \Delta$ cells, although in this case, the presence of native modifications served to modestly reduce methylation activity on several tRNA species that are substrates for ScTrm10 in vitro, but are not normally modified in vivo (Swinehart et al. 2013). Thus, in the S. cerevisiae example, the presence of native modifications may restrict enzyme activity in vivo to only a limited number of tRNA, rather than stimulate catalytic efficiency as we might speculate for hTRMT10B. Again, understanding the nature of hTRMT10B interaction with tRNA and how modifications might affect this interaction would make this more clear. Alternatively, the possibility cannot be excluded that hTRMT10B may require an additional interacting partner, such as another protein, for efficient methylation activity. Several other tRNA modification enzymes have been demonstrated to require partner proteins for catalysis (Vilardo et al. 2012; Guy and Phizicky 2014), although this has not yet been observed for cytosolic Trm10 enzymes, which notably function as monomers instead of the homodimers typical of other SPOUT methyltransferases (Kempenaers et al. 2010; Shao et al. 2014; Van Laer et al. 2016; Hori 2017; Singh et al. 2018; Krishnamohan and Jackman 2019). If hTRMT10B utilizes such a protein partner, it would suggest greater divergence from the cytosolic ScTrm10 and hTRMT10A, and some similarity to the mitochondrial hTRMT10C, with its $\mathrm{m}^{1} \mathrm{G}_{9} / \mathrm{m}^{1} \mathrm{~A}_{9}$ activity and requisite cofactor SDR5C1 (Vilardo et al. 2012). Future biochemical characterization of hTRMT10B, including determinants of its substrate specificity and interactions with other cellular molecules, is needed to further delineate its role from hTRMT10A and hTRMT10C, as well as more completely understand the evolutionary basis for the distinct conserved presence of TRMT10B homologs in vertebrates.

hTRMT10A catalyzes $\mathrm{m}^{1} \mathrm{G}_{9}$ methylation in vitro, consistent with its previously identified function in $\beta$-cells (Cosentino et al. 2018). That study identified three human tRNAs as in vitro and in vivo substrates of hTRMT10A: tRNA $^{\text {Gln(UUG) }}$ tRNA ${ }^{\mathrm{Gln}(\mathrm{CUG})}$, and tRNA ${ }^{\text {iMet }}$. Similarly, Vilardo and colleagues found that hTRMT10A was able to catalyze $\mathrm{m}^{1} \mathrm{G}_{9}$ on one human cytoplasmic (tRNA ${ }^{\mathrm{Arg}}$ )

TABLE 2. Observed single-turnover rates of methylation by Trm10 enzymes

\begin{tabular}{lccc}
\hline Activity & $\begin{array}{c}\text { tRNA species } \\
\text { (anticodon) }\end{array}$ & $\begin{array}{c}\text { hTRMT10A } \\
k_{\text {obs }}\left(\mathrm{min}^{-1}\right)\end{array}$ & $\begin{array}{c}\text { hTRMT10B } \\
k_{\text {obs }}\left(\mathrm{min}^{-1}\right)\end{array}$ \\
\hline $\mathrm{m}^{1} \mathrm{G}_{9}$ & Arg (UCG) & $2.59 \pm 0.54$ & $(6.8 \pm 2.7) \times 10^{-3} \mathrm{a}$ \\
& $\operatorname{Trp}(\mathrm{CCA})$ & $5.21 \pm 1.27$ & $(4.7 \pm 2.4) \times 10^{-3} \mathrm{a}$ \\
$\mathrm{m}^{1} \mathrm{~A}_{9}$ & Asp (GUC) & $\mathrm{ND}^{\mathrm{b}}$ & $(2.5 \pm 1.3) \times 10^{-2}$ \\
\hline
\end{tabular}

${ }^{a}$ Estimate for $k_{\text {obs }}$ derived using the method of linear initial rates. ${ }^{{ }} \mathrm{m}^{1} \mathrm{~A}$ a activity of hTRMT10A was not detected under any experimental conditions tested. 
and three mitochondrial (tRNA ${ }^{\text {lle }}$, tRNA ${ }^{\text {Leu }}$, and tRNA $^{\text {Tyr }}$ ) tRNAs, as well as $\mathrm{m}^{1} \mathrm{l}$ g on a tRNA ${ }^{\text {ArgG9l }}$ variant (Vilardo et al. 2018). Here we confirm the ability of hTRMT10A to methylate tRNA ${ }^{\text {Arg }}$, tRNA ${ }^{\mathrm{Gln}(U \cup G)}$, tRNA ${ }^{\mathrm{Gln}(\mathrm{CUG})}$, and tRNA ${ }^{\text {iMet }}$ in vitro, and add four human tRNAs (tRNA ${ }^{\text {Asn }}$, tRNA $^{\text {Gly(CCC) }}$ tRNA $^{\text {Gly(GCC) }}$, tRNA $\left.{ }^{\text {Trp }}\right)$ and five yeast tRNAs (tRNA $^{\text {Asn }}$ tRNA $^{\text {Gly(CCC) }}$ tRNA $^{\text {Gly(GCC) }}$ tRNA $^{\text {Trp }}, t^{2}$ tRA $^{\text {Val }}$ ) to its repertoire (Table 1; Fig. 2). Thus we demonstrate not only that hTRMT10A is the de facto human $\mathrm{m}^{1} \mathrm{G}_{9}$ methyltransferase, but also that it has a broad capability to methylate many tRNAs, even ones not expressed in human cells. Notably, we observe no methylation of any $A$ residue in our uniformly labeled transcript of tRNA ${ }^{\text {Phe }}$ (Fig. 4), which rules out the recently suggested possibility that hTRMT10B is responsible for the $\mathrm{m}^{1} \mathrm{~A}_{14}$ modification in that tRNA. The enzyme responsible for catalyzing that modification thus remains to be identified (de CrécyLagard et al. 2019).

Of 19 tRNAs that have been identified as having $\mathrm{m}^{1} \mathrm{G}_{9}$ in human cells, six have now been confirmed as substrates of hTRMT10A (tRNA ${ }^{\text {Asn }}$ and tRNA ${ }^{\text {Trp }}$ are added in this current study) (Clark et al. 2016). Interestingly, however, we identify tRNA ${ }^{\mathrm{Gly}(\mathrm{CCC})}$ and tRNA ${ }^{\mathrm{Gly}(\mathrm{GCC})}$ as robust in vitro substrates of hTRMT10A (Table 2), even though they had no detectable levels of $\mathrm{m}^{1} \mathrm{G}_{9}$ methylation in vivo (this does not appear to be a technical issue, as both were successfully identified as having the $\mathrm{m}^{1} \mathrm{~A}_{58}$ modification) (Clark et al. 2016). This echoes the in vitro substrate expansion that has been previously demonstrated for ScTrm10, underscoring further similarities between these two enzymes (Swinehart et al. 2013). Although it has not been determined, it is possible that this substrate expansion is characteristic of the homolog most similar to ScTrm10 (hTRMT10A in humans), and that Trm10 homologs in other organisms will display similar patterns of substrate specificity. Likewise, determining the basis for such divergence between substrate specificity in vitro and in vivo remains an interesting and unanswered question.

\section{MATERIALS AND METHODS}

\section{Saccharomyces cerevisiae complementation}

A previously described trm10 10 strain of S. cerevisiae (Jackman et al. 2003) was transformed with $2 \mu$ (high copy) LEU2 plasmids expressing the indicated Trm10 genes (ScTrm10, hTRMT10A, hTRMT10B, or empty vector) under the control of a galactoseinducible promoter (Swinehart et al. 2013). Cultures of Leu+ transformants were standardized to $\mathrm{OD}_{600}=1$, and tested for 5fluorouracil (5-FU) sensitivity by spotting $2 \mu \mathrm{L}$ of fivefold serial dilutions onto $\mathrm{S}-\mathrm{Gal}-\mathrm{leu}$ plates containing varied concentrations of $5-\mathrm{FU}(0,0.1,1 \mu \mathrm{g} / \mathrm{mL})$. Plates were grown at 30 or $37^{\circ} \mathrm{C}$ for $2-$ $3 \mathrm{~d}$ before being photographed. Western blots were performed on crude lysates prepared as described in Gelperin et al. (2005), and used anti-HA primary antibodies (Abcam, ab18181) and anti-mouse secondary antibodies (Sigma, A9044) to probe for expression of enzymes with amino-terminal HA epitopes. Antibodies were detected using Amersham ECL Western Blotting Detection Reagent (GE Healthcare), and imaging was performed with the Konica SRX-101A developer (Tokyo, Japan).

\section{Expression, purification, and mutagenesis of hTRMT10A and hTRMT10B}

Amino-terminal His 6 -tagged constructs of ScTrm10, hTRMT10A, and TkTrm10 were expressed in E. coli and purified using metal ion affinity chromatography as described previously (Jackman et al. 2003; Swinehart et al. 2013; Krishnamohan et al. 2019). hTRMT10B was expressed and purified using an identical protocol, except that an additional Superdex-200 column (GE Healthcare) was used to improve purification by removal of additional contaminating proteins that remained after metal ion affinity purification, likely due to lower overall levels of expression of hTRMT10B relative to the high-expressing ScTrm10 and hTRMT10A. Gel filtration was performed in a buffer containing $10 \%$ glycerol, $20 \mathrm{mM}$ Bis-tris $\mathrm{pH} 6.5,500 \mathrm{mM} \mathrm{NaCl}, \mathrm{MgCl}$, EDTA, and DTT at $0.4 \mathrm{~mL} / \mathrm{min}$, with hTRMT10B-containing fractions (identified by SDS-PAGE) pooled and stored at $-80^{\circ} \mathrm{C}$ in the same buffer. The resulting proteins were judged to be $75 \%-90 \%$ pure by SDS-PAGE. hTRMT10B variants were generated using Phusion mutagenesis (Thermo Fisher Scientific) and verified by DNA sequencing; variant proteins were purified as described above for the wild-type enzyme but without the additional size exclusion purification step, as further purification was not needed to detect activity under the tested conditions (Swinehart et al. 2013).

\section{In vitro methylation assays}

In vitro $\mathrm{m}^{1} \mathrm{R}_{9}$ methylation assays were performed using uniformly labeled in vitro transcripts as previously described (Swinehart et al. 2013). Briefly, inclusion of either [ $\alpha^{32}$ P]-GTP (for visualization of $G_{9}$ methylation activity) or [ $\left.\alpha^{32} \mathrm{P}\right]$-ATP (for visualization of $A_{9}$ methylation activity) in T7 RNA polymerase in vitro transcription reactions were used to generate all substrate tRNAs. No additional refolding step was used prior to assays, based on previous observations that these did not affect the extent or rate of methylation of these substrate tRNA transcripts. Methylation reactions contained 2000 counts per minute of the indicated tRNA, 50 $\mathrm{mM}$ Tris $\mathrm{pH}$ 8.0, $1.5 \mathrm{mM} \mathrm{MgCl}_{2}$ and $0.5 \mathrm{mM} \mathrm{SAM}$, and were initiated by the addition of enzyme (fivefold serial dilutions starting with the highest possible concentrations of each enzyme: $2 \mathrm{mg} /$ $\mathrm{mL}$ for ScTrm10, $1.5 \mathrm{mg} / \mathrm{mL}$ for hTRMT10A, $0.3 \mathrm{mg} / \mathrm{mL}$ for hTRMT10B, $0.3 \mathrm{mg} / \mathrm{mL}$ for hTRMT10B G231R, and $0.2 \mathrm{mg} / \mathrm{mL}$ for hTRMT10B G231R/G232R). After incubation for $1 \mathrm{~h}$ at $37^{\circ} \mathrm{C}$, reactions were quenched by addition of phenol: chloroform: isoamyl alcohol (25:24:1) and purified by phenol extraction and ethanol precipitation. The purified tRNA was then digested to single nucleotides using nuclease P1 (Sigma-Aldrich) leaving the $5^{\prime}{ }_{-}^{32} \mathrm{P}$ label on methylated $\left(p^{*} m^{1} G / A\right)$ or unmethylated $\left(p^{*} G / A\right) G$ or $A$ residues, which were resolved by cellulose TLC in isobutyric acid: $\mathrm{H}_{2} \mathrm{O}: \mathrm{NH}_{4} \mathrm{OH}$ (66:33:1; $\mathrm{G}$ residues) or saturated ammonium sulfate: $\mathrm{H}_{2} \mathrm{O}$ : isopropanol (80:18:2; A residues) solvent. The plates were exposed to a phosphor screen and scanned using the 
Typhoon imaging system (GE Healthcare) and quantified using ImageQuant TL software (GE Healthcare).

Single-turnover assays contained the same uniformly labeled transcripts and were performed under the same reaction conditions used for the activity assays above, but included $\geq 2 \mu \mathrm{M}$ of each enzyme (to achieve $>100$-fold excess over concentration of tRNA). Aliquots were taken at time points (0.3-90 min) and quenched with phenol: chloroform: isoamyl alcohol (25:24:1), purified, digested and analyzed by TLC as described above. The percent $m^{1} G_{9} / m^{1} A_{9}(\% P)$ formed at each time point was quantified and plotted as a function of time $(t)$, and $k_{\text {obs }}$ was determined by fitting to a single exponential (Equation 1) or linear (Equation 2) equation using Kaleidagraph software (Synergy Software); the method of initial rates was used to calculate $k_{\text {obs }}$ for fits with a linear initial velocity.

$$
\begin{gathered}
\% P=P_{\max }{ }^{*}\left(1-\exp \left(-k_{\mathrm{obs}}{ }^{*} t\right)\right) \\
\% P=\left(\left(k_{\mathrm{obs}}{ }^{*} P_{\max }\right)^{*} t\right)+P_{\min }
\end{gathered}
$$

Rates were all measured independently using at least two different excess enzyme concentrations for each enzyme and did not depend on the concentration of enzyme in the assays, as expected for the maximal enzyme concentration-independent rate that is limiting for the overall chemistry of methylation (Krishnamohan and Jackman 2017).

\section{HPLC-MS/MS analysis of nucleosides and oligonucleotides}

One microgram aliquots of tRNA ${ }^{\text {Asp }}$ transcript (with and without hTRMT10B in vitro incubation) were hydrolyzed to nucleosides using nuclease P1 and alkaline phosphatase as previously described (Dumelin et al. 2012) or oligonucleotides using ribonuclease T1 and alkaline phosphatase as previously described (Addepalli and Limbach 2016). For nucleosides, HPLC-MS/MS analyses were carried out on an Orbitrap Fusion Lumos Tribrid mass spectrometer (Thermo Fisher Scientific, Waltham, MA) coupled to a Vanquish Flex Quaternary (Thermo Fisher Scientific, Waltham, MA) UHPLC system in a similar fashion as previously described (Jora et al. 2018). An Acquity UPLC HSS T3 column, 1.8 $\mu \mathrm{m}, 1.0 \mathrm{~mm} \times 100 \mathrm{~mm}$ (Waters) was used. The mobile phase buffers were prepared from $5.3 \mathrm{mM}$ ammonium acetate at $\mathrm{pH} 4.5$ in water (mobile phase A) and in 40/60 acetonitrile/water (mobile phase B). The reversed-phase HPLC gradient consisted of $0 \% \mathrm{~B}$ for $7.6 \mathrm{~min}, 2 \% \mathrm{~B}$ at $15.7 \mathrm{~min}, 3 \% \mathrm{~B}$ at $19.2 \mathrm{~min}, 5 \% \mathrm{~B}$ at 25.7 $\mathrm{min}, 25 \% \mathrm{~B}$ at $29.5 \mathrm{~min}, 50 \% \mathrm{~B}$ at $32.3 \mathrm{~min}, 75 \% \mathrm{~B}$ at $36.4 \mathrm{~min}$ (hold for $0.2 \mathrm{~min}$ ), $99 \% \mathrm{~B}$ at $39.6 \mathrm{~min}$ (hold for $7.2 \mathrm{~min}$ ), then returning to $0 \% \mathrm{~B}$ at $46.9 \mathrm{~min}$. An 18-min step at $0 \%$ B was used for column reequilibration. Flow rate and column temperature were set at $100 \mu \mathrm{L} \mathrm{min}^{-1}$ and $30^{\circ} \mathrm{C}$, respectively.

For oligonucleotides, HPLC-MS/MS analyses were done using the same system described above, and under previously described conditions (Lobue et al. 2019a,b). A PEEK Shodex HILICpak VN-50 column, $5 \mu \mathrm{m}, 2.0 \mathrm{~mm} \times 150 \mathrm{~mm}$ (Shoko Science) was used. Mobile phases A and B consisted of, respectively, $70 \mathrm{mM}$ ammonium acetate (no $\mathrm{pH}$ control) in 30/70 acetonitrile/water and $25 \mathrm{mM}$ ammonium acetate (no pH control) in 75/ 25 acetonitrile/water. The hydrophilic interaction liquid chromatography (HILIC) HPLC gradient consisted of $100 \%$ B for 1.0 $\min , 45 \% \mathrm{~B}$ at $30 \mathrm{~min}$, then returning to $100 \% \mathrm{~B}$ at $30.1 \mathrm{~min}$. $A$ 25 -min reequilibration step at $100 \% B$ was used. Data processing was performed using RNAModMapper (Yu et al. 2017; Lobue et al. 2019b) with the following settings: variable mapping mode; precursor and product mass tolerances set to $0.06 \mathrm{~m} / \mathrm{z}_{\text {; }}$ $c / y$-type ions weighting factor at 0.7 ; and $P$-score and Dot product thresholds at 55 and 0.8 , respectively.

\section{Filter binding assays}

Uniformly labeled transcripts were incubated with varied concentrations of hTRMT10A and hTRMT10B (from $10 \mathrm{nM}$ to $10 \mu \mathrm{M}$ ) for at least $15 \mathrm{~min}$, at which point the reactions were vacuum filtered through Amersham Protran $0.45 \mu \mathrm{m}$ nictrocellulose membrane and Amersham Hybond- $\mathrm{N}^{+}$membrane, in sequence, followed by washing with buffer using a dot-blot apparatus. Each membrane was dried separately, exposed to a phosphor screen and imaged using Typhoon imaging system (GE Healthcare), and quantified using ImageQuant TL software (GE Healthcare). The fraction of tRNA bound (\%B) was plotted as a function of enzyme concentration, and $K_{D}$ was determined by fitting to a binding isotherm, where $\% B=$ fraction of bound tRNA, $B_{\max }=$ maximal fraction of bound tRNA, $B_{\min }=$ minimal fraction of bound tRNA, $[E]=$ enzyme concentration, $K_{D}=$ apparent dissociation constant, and $H=$ cooperativity coefficient (Equation 3 ).

$$
\% B=\% B_{\max }+\frac{\% B_{\min }-\% B_{\max }}{1+\frac{[E]}{K_{D}}}
$$

\section{SUPPLEMENTAL MATERIAL}

Supplemental material is available for this article.

\section{ACKNOWLEDGMENTS}

The authors thank Dylan Fortman for construction of constructs used for yeast complementation assays. This work was supported by National Institutes of Health (NIH) GM130135 to J.E.J. and National Science Foundation (NSF) CHE1507357 and $\mathrm{NIH}$ OD018485 to P.A.L. Research reported in this publication was also supported by the Office of the Director, National Institutes of Health under award number S10OD023582.

Received May 24, 2019; accepted July 7, 2019.

\section{REFERENCES}

Abbott JA, Francklyn CS, Robey-Bond SM. 2014. Transfer RNA and human disease. Front Genet 5: 158. doi:10.3389/fgene.2014 .00158

Addepalli B, Limbach PA. 2016. Pseudouridine in the anticodon of Escherichia colitRNA ${ }^{\text {Tyr( }(\mathrm{Q} \Psi \mathrm{A})}$ is catalyzed by the dual specificity enzyme RluF. J Biol Chem 291: 22327-22337. doi:10.1074/jbc M116.747865

Clark WC, Evans ME, Dominissini D, Zheng G, Pan T. 2016. tRNA base methylation identification and quantification via high-throughput sequencing. RNA 22: 1771-1784. doi:10.1261/rna.056531.116

Cosentino C, Toivonen S, Diaz Villamil E, Atta M, Ravanat JL, Demine S, Schiavo AA, Pachera N, Deglasse JP, Jonas JC, et al. 
2018. Pancreatic $\beta$-cell tRNA hypomethylation and fragmentation link TRMT10A deficiency with diabetes. Nucleic Acids Res 46: 10302-10318. doi:10.1093/nar/gky839

de Crécy-Lagard V, Boccaletto P, Mangleburg CG, Sharma P, Lowe TM, Leidel SA, Bujnicki JM. 2019. Matching tRNA modifications in humans to their known and predicted enzymes. Nucleic Acids Res 47: 2143-2159. doi:10.1093/nar/gkz011

Dumelin CE, Chen Y, Leconte AM, Chen YG, Liu DR. 2012. Discovery and biological characterization of geranylated RNA in bacteria. Nat Chem Biol 8: 913. doi:10.1038/nchembio.1070

Endres L, Dedon PC, Begley TJ. 2015. Codon-biased translation can be regulated by wobble-base tRNA modification systems during cellular stress responses. RNA Biol 12: 603-614. doi:10.1080/ 15476286.2015.1031947

Gelperin DM, White MA, Wilkinson ML, Kon Y, Kung LA, Wise KJ, Lopez-Hoyo N, Jiang L, Piccirillo S, Yu H, et al. 2005. Biochemical and genetic analysis of the yeast proteome with a movable ORF collection. Genes Dev 19: 2816-2826. doi:10 $.1101 /$ gad.1362105

Gillis D, Krishnamohan A, Yaacov B, Shaag A, Jackman JE, Elpeleg O. 2014. TRMT10A dysfunction is associated with abnormalities in glucose homeostasis, short stature and microcephaly. J Med Genet 51: 581-586. doi:10.1136/jmedgenet-2014-102282

Gu C, Begley TJ, Dedon PC. 2014. tRNA modifications regulate translation during cellular stress. FEBS Lett 588: 4287-4296. doi:10 .1016/j.febslet.2014.09.038

Gustavsson M, Ronne H. 2008. Evidence that tRNA modifying enzymes are important in vivo targets for 5-fluorouracil in yeast. RNA 14: 666-674. doi:10.1261/rna.966208

Guy MP, Phizicky EM. 2014. Two-subunit enzymes involved in eukaryotic post-transcriptional tRNA modification. RNA Biol 11: 16081618. doi:10.1080/15476286.2015.1008360

Hori H. 2017. Transfer RNA methyltransferases with a SpoU-TrmD (SPOUT) fold and their modified nucleosides in tRNA. Biomolecules 7: E23. doi:10.3390/biom7010023

Igoillo-Esteve M, Genin A, Lambert N, Désir J, Pirson I, Abdulkarim B, Simonis N, Drielsma A, Marselli L, Marchetti P, et al. 2013. tRNA methyltransferase homolog gene TRMT10A mutation in young onset diabetes and primary microcephaly in humans. PLoS Genet 9: e1003888. doi:10.1371/journal.pgen.1003888

Jackman JE, Alfonzo JD. 2013. Transfer RNA modifications: nature's combinatorial chemistry playground. Wiley Interdiscip Rev RNA 4: 35-48. doi:10.1002/wrna.1144

Jackman JE, Montange RK, Malik HS, Phizicky EM. 2003. Identification of the yeast gene encoding the tRNA m1G methyltransferase responsible for modification at position 9. RNA 9: 574-585. doi:10.1261/rna.5070303

Jora M, Burns AP, Ross RL, Lobue PA, Zhao R, Palumbo CM, Beal PA, Addepalli B, Limbach PA. 2018. Differentiating positional isomers of nucleoside modifications by higher-energy collisional dissociation mass spectrometry (HCD MS). J Am Soc Mass Spectrom 29: 1745-1756. doi:10.1007/s13361-018-1999-6

Juhling F, Morl M, Hartmann RK, Sprinzl M, Stadler PF, Putz J. 2009. tRNAdb 2009: compilation of tRNA sequences and tRNA genes. Nucleic Acids Res 37: D159-162. doi:10.1093/nar/gkn772

Kempenaers M, Roovers M, Oudjama Y, Tkaczuk KL, Bujnicki JM, Droogmans L. 2010. New archaeal methyltransferases forming 1methyladenosine or 1-methyladenosine and 1-methylguanosine at position 9 of tRNA. Nucleic Acids Res 38: 6533-6543. doi:10 $.1093 / \mathrm{nar} / \mathrm{gkq} 451$

Kirchner S, Ignatova Z. 2015. Emerging roles of tRNA in adaptive translation, signalling dynamics and disease. Nat Rev Genet 16: 98-112. doi:10.1038/nrg3861
Krishnamohan A, Jackman JE. 2017. Mechanistic features of the atypical tRNA $m^{1} \mathrm{G}_{9}$ SPOUT methyltransferase, Trm10. Nucleic Acids Res 45: 9019-9029. doi:10.1093/nar/gkx620

Krishnamohan A, Jackman JE. 2019. A family divided: distinct structural and mechanistic features of the SpoU-TrmD (SPOUT) methyltransferase superfamily. Biochemistry 58: 336-345. doi:10.1021/ acs.biochem.8b01047

Krishnamohan A, Dodbele S, Jackman JE. 2019. Insights into catalytic and tRNA recognition mechanism of the dual-specific tRNA methyltransferase from Thermococcus kodakarensis. Genes (Basel) 10: E100. doi:10.3390/genes10020100

Lobue PA, Jora M, Addepalli B, Limbach PA. 2019a. Oligonucleotide analysis by hydrophilic interaction liquid chromatography-mass spectrometry in the absence of ion-pair reagents. J Chromatogr A 1595: 39-48. doi:10.1016/j.chroma.2019.02.016

Lobue PA, Yu N, Jora M, Abernathy S, Limbach PA. 2019b. Improved application of RNAModMapper-an RNA modification mapping software tool-for analysis of liquid chromatography tandem mass spectrometry (LC-MS/MS) data. Methods 156: 128-138. doi:10.1016/j.ymeth.2018.10.012

Machnicka MA, Olchowik A, Grosjean H, Bujnicki JM. 2014. Distribution and frequencies of post-transcriptional modifications in tRNAs. RNA Biol 11: 1619-1629. doi:10.4161/15476286.2014 .992273

Phizicky EM, Hopper AK. 2010. tRNA biology charges to the front. Genes Dev 24: 1832-1860. doi:10.1101/gad.1956510

Shao Z, Yan W, Peng J, Zuo X, Zou Y, Li F, Gong D, Ma R, Wu J, Shi Y, et al. 2014. Crystal structure of tRNA $\mathrm{m}^{1} \mathrm{G}_{9}$ methyltransferase Trm 10: insight into the catalytic mechanism and recognition of tRNA substrate. Nucleic Acids Res 42: 509-525. doi:10.1093/ nar/gkt869

Sievers F, Wilm A, Dineen D, Gibson TJ, Karplus K, Li W, Lopez R, McWilliam H, Remmert M, Soding J, et al. 2011. Fast, scalable generation of high-quality protein multiple sequence alignments using Clustal Omega. Mol Syst Biol 7: 539. doi:10.1038/msb .2011 .75

Singh RK, Feller A, Roovers M, Van Elder D, Wauters L, Droogmans L, Versées W. 2018. Structural and biochemical analysis of the dualspecificity Trm10 enzyme from Thermococcus kodakaraensis prompts reconsideration of its catalytic mechanism. RNA 24: 1080-1092. doi:10.1261/rna.064345.117

Swinehart WE, Henderson JC, Jackman JE. 2013. Unexpected expansion of tRNA substrate recognition by the yeast $\mathrm{m}^{1} \mathrm{G}_{9}$ methyltransferase Trm10. RNA 19: 1137-1146. doi:10.1261/rna.039651.113

Tuorto F, Lyko F. 2016. Genome recoding by tRNA modifications. Open Biol 6: 160287. doi:10.1098/rsob.160287

Van Laer B, Roovers M, Wauters L, Kasprzak JM, Dyzma M, Deyaert E, Kumar Singh R, Feller A, Bujnicki JM, Droogmans L, et al. 2016. Structural and functional insights into tRNA binding and adenosine N1-methylation by an archaeal Trm10 homologue. Nucleic Acids Res 44: 940-953. doi:10.1093/nar/gkv1369

Vilardo E, Nachbagauer C, Buzet A, Taschner A, Holzmann J, Rossmanith W. 2012. A subcomplex of human mitochondrial RNase $\mathrm{P}$ is a bifunctional methyltransferase-extensive moonlighting in mitochondrial tRNA biogenesis. Nucleic Acids Res 40: 11583-11593. doi:10.1093/nar/gks910

Vilardo E, Nachbagauer C, Buzet A, Taschner A, Holzmann J, Rossmanith W. 2018. A subcomplex of human mitochondrial RNase $\mathrm{P}$ is a bifunctional methyltransferase-extensive moonlighting in mitochondrial tRNA biogenesis. Nucleic Acids Res 46: 11126-11127. doi:10.1093/nar/gky931

Yu N, Lobue PA, Cao X, Limbach PA. 2017. RNAModMapper: RNA modification mapping software for analysis of liquid chromatography tandem mass spectrometry data. Anal Chem 89: 1074410752. doi:10.1021/acs.analchem.7b01780 

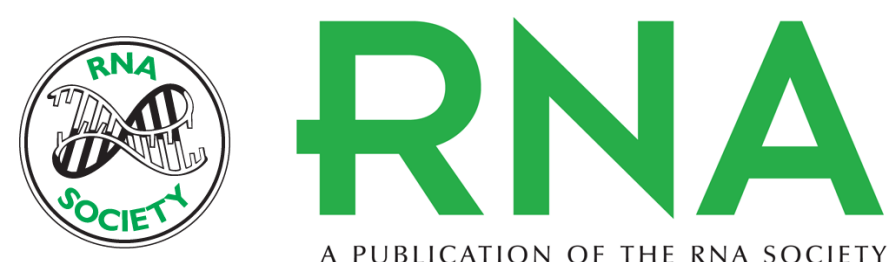

A PUBLICATION OF THE RNA SOCIETY

\section{Distinct substrate specificities of the human tRNA methyltransferases TRMT10A and TRMT10B}

Nathan W. Howell, Manasses Jora, Benjamin F. Jepson, et al.

RNA 2019 25: 1366-1376 originally published online July 10, 2019

Access the most recent version at doi:10.1261/rna.072090.119

\section{Supplemental http://rnajournal.cshlp.org/content/suppl/2019/07/10/rna.072090.119.DC1 Material}

References This article cites 36 articles, 9 of which can be accessed free at: http://rnajournal.cshlp.org/content/25/10/1366.full.html\#ref-list-1

Creative This article is distributed exclusively by the RNA Society for the first 12 months after the Commons License full-issue publication date (see http://rnajournal.cshlp.org/site/misc/terms.xhtml). After 12 months, it is available under a Creative Commons License (Attribution-NonCommercial 4.0 International), as described at http://creativecommons.org/licenses/by-nc/4.0/.

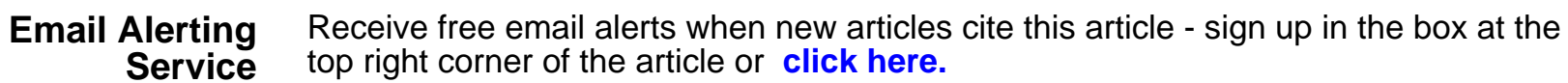

